\title{
A Funcionalidade e Qualidade de Vida em Crianças com Paralisia Cerebral Bilateral e Unilateral
}

\author{
Luise Ferreira de Queiroz, ${ }^{1}$ Miriam Cabrera Corvelo Delboni, ${ }^{1}$ \\ Marciane Montagner Missio, ${ }^{2}$ Claudia Morais Trevisan ${ }^{1}$
}

\begin{abstract}
RESUMO
Objetivo: O estudo buscou avaliar a funcionalidade e qualidade de vida de crianças com paralisia cerebral (PC) de acordo com a distribuição anatômica baseado no componente atividade e participação da Classificação Internacional de Funcionalidade, Incapacidade e Saúde. Método: Estudo transversal, do tipo descritivo, com levantamento de dados por meio da Medida de Função Motora Grossa, Inventário de Avaliação Pediátrica de Incapacidade e Questionário de Qualidade de Vida de Crianças. A análise dos dados foi por estatística descritiva, sendo apresentados em média e desvio padrão. A amostra por conveniência do estudo foi composta por crianças com diagnóstico de paralisia cerebral bilateral e unilateral. Resultados: No estudo $52 \%$ $(n=10)$ possuíam paralisia unilateral e $48 \%(n=9)$ bilateral. No instrumento Medida de Função, a pontuação não obteve variação significativa para o tipo de paralisia. No Inventário as crianças com paralisia bilateral alcançaram escores superiores nas habilidades funcionais e inferiores na área de assistência do cuidador. O Questionário evidenciou que as crianças com paralisia bilateral têm maior qualidade de vida que as com paralisia unilateral. A distribuição anatômica da paralisia em bilateral e unilateral não foi determinante para definir alterações na funcionalidade. Os fatores contextuais (físico, social e atitudinal) manifestaram influência substancial, pois condições físicas do ambiente e atitudes dos cuidadores surgiram como principais alterações. Conclusões: O estudo permitiu uma análise global da funcionalidade e da qualidade de vida na PC, em que crianças com PC bilateral aceitam melhor sua condição física e as crianças com PC unilateral tendem a negligenciar o hemicorpo, prejudicando suas próprias habilidades funcionais.
\end{abstract}

Palavras-chave: Paralisia cerebral. Terapia ocupacional. Atividades cotidianas. Participação social.

FUNCTIONALITY AND QUALITY OF LIFE IN CHILDREN WITH BILATERAL AND UNILATERAL CEREBRAL PALSY

ABSTRACT

Objective: The study sought to assess the functionality and quality of life of children with Cerebral Palsy according to the anatomical distribution based on the activity and participation component of the International Classification of Functionality, Disability and Health. Methods: Cross-sectional, descriptive study, with survey of data through the Gross Motor Function Measure, Pediatric Disability Assessment Inventory and Children's Quality of Life Questionnaire. Data analysis was performed using descriptive statistics, presented as mean and standard deviation. The convenience sample of the study was composed of children diagnosed with bilateral and unilateral Cerebral Palsy. Results: In the study $52 \%(n=10)$ had unilateral paralysis and $48 \%(n=9)$ bilateral. In the Function Measure instrument, the score did not show significant variation for the type of paralysis. In the Inventory, children with bilateral paralysis achieved higher scores on functional skills and lower scores in the caregiver's care area. The Questionnaire showed that children with bilateral paralysis have a higher quality of life than those with unilateral paralysis. The anatomical distribution of Paralysis in bilateral and unilateral was not decisive to define changes in functionality. The contextual factors (physical, social and attitudinal) showed substantial influence, since physical conditions of the environment and attitudes of caregivers emerged as the main changes. Conclusions: The study allowed a global analysis of functionality and quality of life in CP, where children with bilateral CP accept better their physical condition and children with unilateral CP tend to neglect the hemibody, impairing their own functional abilities.

Keywords: Cerebral palsy. Occupational therapy. Activities of daily living. Social participation.

RECEBIDO EM: 15/12/2019

MODIFICAÇÕES SOLICITADAS EM: 22/2/2020

ACEITO EM: 2/5/2020

\footnotetext{
Universidade Federal de Santa Maria - Santa Maria/RS, Brasil.

Autora correspondente. Universidade Federal de Santa Maria. Rua Tapes, Itararé, Santa Maria/RS, Brasil. http://lattes.cnpq.br/4050653991858988. https://orcid.org/0000-0002-2621-4547. marcimissio@gmail.com
} 


\section{INTRODUÇÃO}

A paralisia cerebral $(P C)$ é conceituada para além de um distúrbio de posturas e movimentos, frequentemente está associada a uma série de deficiências cognitivas, linguísticas, comunicativas, sensoriais ou perceptivas. Os déficits ocasionados pela PC podem afetar além dos componentes cognitivos ou motores, também a combinação entre os dois ou mais desses componentes, desde o desempenho de comando simples como da execução de uma dupla tarefa (FLUSS; LIDZBA, 2020).

$\mathrm{Na}$ literatura científica podem ser encontradas classificações para a PC de acordo com as características clínicas mais predominantes, como a do tipo espástica, discinética, atáxica e mista. A PC espástica é compreendida pelo aumento do tônus muscular causado por lesão no sistema piramidal, manifestando frequentemente alterações na postura, fala e equilíbrio (WAGNER et al., 2020). A PC discinética corresponde a uma lesão extrapiramidal, presença de hipertonia plástica com sinal de roda denteada, movimentos involuntários rápidos e de pequena amplitude em regiões distais e em alguns casos, o opistótono. Já na PC atáxica verificam-se comprometimento cerebelar com alteração de equilíbrio e coordenação motora ao executar movimentos voluntários, dismetria, disartria e sialorreia. Além destas, a PC mista refere-se à combinação do tipo espástico, discinético e atáxico, ocasionando ampla gama de comprometimentos (MENESES, 2019).

A fim de determinar a distribuição anatômica da PC, vem sendo utilizada classificação baseada em diferentes enfoques, o de anormalidade motora que predomina (atáxica, discinética ou espástica), a distribuição topográfica (bilateral ou unilateral para espasticidade) e a funcionalidade (SILVA; DIAS; PFEIFER, 2016). Com tantas formas de apresentação do quadro clínico da PC, entretanto, principalmente quanto aos tipos de alterações, os perfis de funcionalidade manifestam-se de forma individual e são influenciados pelos fatores contextuais.

A Classificação Internacional de Funcionalidade, Incapacidade e Saúde (CIF), compreendida por uma abordagem biopsicossocial, tende a contribuir para a compreensão dos fatores intrínsecos e extrínsecos que podem influenciar na funcionalidade de crianças com PC (SCHIARITI; MÂSSE, 2015). A CIF estabelece uma linguagem clara para descrição de saúde e de seus estados relacionados, possibilitando a todos os profissionais de saúde uma mesma compreensão global (PIEXAK et al., 2019).
A CIF descreve o funcionamento humano e a incapacidade como uma interação entre diversos estados de saúde, de estrutura, função do corpo, atividade e participação em fatores contextuais que consideram a história e o estilo de vida do indivíduo (BRITTO et al., 2018).

Os instrumentos buscam avaliar algumas áreas específicas do desenvolvimento motor, e comumente cada avaliação possui especificidades de interesse de cada profissional conforme o objetivo terapêutico do tratamento. A realização de avaliações mais abrangentes na PC vem se mostrando necessária para propiciar maior conhecimento das habilidades, capacidades e desempenho funcional da criança, além de levar em consideração o seu envolvimento nos diferentes contextos de vida (SOUZA; ALPINO, 2015).

A CIF serve como guia importante para a escolha de instrumentos de mensuração a serem utilizados na definição de objetivos para o tratamento e metas de resultados significativos (DORNELES et al., 2014). O seu modelo pode ser instituído utilizando instrumentos validados previamente para a avaliação de domínios específicos da classificação. Além de permitir a descrição da experiência dos indivíduos, o modelo possibilita uma visão geral dos recursos necessários para melhorar a funcionalidade (BIZ; CHUN, 2019).

Considera-se importante obter uma avaliação global de todas as crianças, independentemente da gravidade do diagnóstico, pressupondo uma concepção de saúde ampliada, em que todas as áreas e capacidades de desempenho sejam consideradas. Desse modo, o objetivo do estudo foi realizar uma avaliação ampliada em crianças com paralisia cerebral bilateral e unilateral utilizando instrumentos avaliativos da funcionalidade e qualidade de vida para analisar os componentes atividade e participação baseados na CIF.

\section{MÉTODOS}

Estudo transversal, do tipo descritivo, realizado com 19 crianças com PC espástica, na faixa etária de 5 a 11 anos, 11 meses e 29 dias (BRASIL, 2005). Foram incluídas no estudo crianças com PC espástica nos níveis I ou II do Sistema de Classificação da Função Motora Grossa (GMFCS). Foram excluídas da amostra crianças submetidas à cirurgia ortopédica em membros inferiores ou superiores, as que aplicaram toxina botulínica nos últimos seis meses ou que apresentassem alterações intelectuais que pudessem comprometer sua participação na pesquisa. 
As crianças foram selecionadas pela pesquisadora por meio de busca ativa na rede municipal e estadual de educação em uma cidade da região central do Rio Grande do Sul, mediante contato com professores municipais e estaduais da área de abrangência. Na amostra por conveniência os participantes do estudo foram divididos de acordo com a distribuição anatômica da deficiência. A coleta de dados foi realizada com todos os participantes em um mesmo local, com duração de 1 hora de entrevista, quando os participantes foram entrevistados juntamente com seus pais ou cuidadores em dia e horário agendados conforme disponibilidade destes e da pesquisadora. No momento da avaliação os participantes não estavam utilizando órteses e todos frequentavam regularmente a escola.

O estudo foi aprovado no Comitê de Ética e Pesquisa com Seres Humanos da Universidade Federal de Santa Maria (UFSM) sob CAAE 12862713.7.0000.5346, emenda 1.525.568 e 1.597.091. Os pais ou responsáveis consentiram com a participação no estudo e as crianças assinaram o Termo de Assentimento, obedecendo aos aspectos éticos da Declaração de Helsinque e do Conselho Nacional de Saúde.

O procedimento usado neste estudo foi o Sistema de Classificação da Função Motora Grossa (GMFCS), utilizado como critério de inclusão do estudo (SILVA; PFEIFER; FUNAYAMA, 2010). Trata-se de uma classificação em cinco níveis, por ordem decrescente, que retrata o nível de independência e habilidades, sendo o nível I andar sem limitações, nível II andar com limitações, nível III andar utilizando um dispositivo manual de mobilidade, nível IV automobilidade com limitações ou mobilidade motorizada e nível $\mathrm{V}$ transportado em uma cadeira de rodas manual (HIRATUKA; MATSUKURA; PFEIFER, 2010).

A escolha dos instrumentos para compor a avaliação foi baseada em uma revisão na literatura de Schiariti et al. (2013), identificando as ferramentas mais utilizadas e consolidadas que mensurassem a funcionalidade de crianças com PC no componente atividade e participação da CIF.

A identificação da criança e seu responsável foi realizada por meio de uma ficha sociodemográfica criada pela pesquisadora para registrar idade, escolaridade, topografia, medicamentos e dados do responsável. Outros instrumentos de avaliação deste estudo foram o Inventário de Avaliação Pediátrica de Incapacidade (Pedi) e o Questionário de Qualidade de Vida de Crianças (CP QOL-Child), ambos respondidos pelos pais ou responsáveis da criança. A mensuração da função motora grossa foi realizada por intermédio da
Medida da Função Motora Grossa (GMFM) a partir da observação das pesquisadoras. $O$ índice de confiança intra e interavaliadores foi Kappa=1.0.

A GMFM traduzida e validada para a população brasileira por Cyrillo e Galvão (2011) foi utilizada para avaliar as capacidades funcionais das crianças. Esta medida foi pontuada a partir da observação das pesquisadoras baseada no melhor desempenho em até três tentativas de realização do comando. Este instrumento é composto por 88 itens divididos em cinco dimensões: deitar e rolar; sentar; engatinhar e ajoelhar; ficar em pé; andar, correr e pular, nas quais a atividade funcional da criança é avaliada em diversas tarefas (CYRILLO; GALVÃO, 2015). O escore (0) corresponde a não inicia a tarefa, (1) inicia, (2) completa parcialmente a tarefa e (3) completa totalmente a tarefa. Estes itens são calculados para cada atividade e posteriormente o cálculo percentual de cada dimensão é realizado. As médias destes valores geram a pontuação total do instrumento.

O Pedi traduzido e validado para a população brasileira foi utilizado na análise do desempenho de tarefas cotidianas realizadas por crianças (MANCINI, 2005). $O$ teste é constituído por três partes: a primeira avalia as habilidades funcionais da criança, sendo pontuado com (0) para incapacidade em desempenhar a atividade e (1) para capacidade de desempenho. A segunda parte do teste mensura a independência da criança para realizar tarefas funcionais, ou seja, a necessidade de assistência do cuidador que a criança requer, sendo a pontuação obtida por meio de uma escala de cinco pontos, em que 5 corresponde à independência, 4 supervisão, 3 assistência mínima, 2 assistência moderada e 1 assistência máxima. A terceira parte do teste informa sobre as modificações do ambiente físico necessárias, sendo pontuada em uma escala nominal de (N) nenhuma modificação, (C) modificação centrada na criança, $(R)$ modificação de reabilitação e (E) modificação extensiva. As três partes do instrumento consideram as áreas de autocuidado, mobilidade e função social.

Neste estudo foram consideradas para análise somente a primeira e a segunda parte do Pedi, pois a terceira faz referência ao domicílio da criança e a entrevista foi realizada com todos pacientes em um mesmo local, que não corresponde ao domicílio da criança (PAVÃO et al., 2014). A faixa etária do teste varia entre 6 meses e 7,5 anos de idade, podendo ser utilizado em crianças com idade superior ao limite indicado, desde que o desempenho funcional esteja dentro da faixa etária (MANCINI, 2005). A base de referência para escolha da faixa etária foi a de Mancini (2005). 
A interpretação dos dados obtidos pelo Pedi ocorre por meio da transformação dos escores brutos em escores padronizados normativos e escores padronizados contínuos. Devido à faixa etária do estudo, no entanto, foi necessário que os resultados fossem analisados somente por intermédio dos escores padronizados contínuos.

O Questionário de Qualidade de Vida de Crianças (CP QOL-Child), versão para o cuidador, traduzido e validado para a população brasileira no estudo de Braccialli et al. (2013), avaliou a percepção dos pais quanto à qualidade de vida das crianças de 4 a 12 anos em alguns domínios, incluindo bem-estar social e aceitação, funcionalidade, participação e saúde física, bem-estar emocional e autoestima, acesso a serviços, dor e impacto da deficiência e saúde da família (DAVIS et al., 2010).

O CP QOL-Child é composto por 66 questionamentos pontuados de 1 a 9 pontos, sendo o escore mínimo (1) e o escore máximo (9), em que as respostam variam entre muito triste (escore mínimo) até muito feliz (escore máximo) para os questionamentos 1 a 43 e a pergunta 52; para as questões 44 até 46 os escores variam entre não fica chateado até fica muito chateado. Na questão 47 a pontuação é diferenciada em uma escala de até cinco pontos, que consiste em (1) nunca, (2) raramente, (3) às vezes, (4) quase sempre e (5) sempre. Na porção final do questionário as respostas são diferenciadas: na questão 48 (não muito preocupado até muito preocupado); questão 49 (não sente dor até muita dor), questão 50 (não incomodado até muito incomodado); questão 51 (não sente desconforto até sente muito desconforto). Para cada domínio do questionário é realizado o cálculo da média.
O Pedi e o GMFM foram utilizados para avaliar o desempenho das crianças por considerar que apenas um deles não contemplaria todos os aspectos dos componentes atividade e participação da CIF. Os resultados dos instrumentos GMFM e CP QOL-Child estão expostos em percentual e o Pedi em escore contínuo. Foi realizada estatística descritiva dos dados e estes estão apresentados em média e desvio padrão.

\section{RESULTADOS}

A participação do estudo teve 19 crianças com paralisia cerebral do tipo espástica, divididas em grupo unilateral e bilateral, de acordo com o comprometimento da paralisia cerebral. Somente foram incluídas crianças com classificação I e II no sistema GMFCS; quando submetidas à cirurgia ortopédica ou toxina botulínica nos últimos seis meses, estas foram excluídas para não subestimar a capacidade das crianças que estariam em processo de adaptação à cirurgia e à toxina.

As características sociodemográficas destacaram na Tabela 1 a predominância de crianças do sexo masculino, com uma média de idade em torno de 8 a 9 anos de idade.

$\mathrm{Na}$ avaliação GMFM as tarefas motoras nas suas 5 dimensões: deitar e rolar, sentar, engatinhar e ajoeIhar, ficar em pé, andar, correr e pular estão apresentadas na Tabela 2. Os dados coletados apontaram que apenas nas dimensões $C$ (engatinhar e ajoelhar), D (ficar em pé) e $E$ (andar, correr e pular) a função motora grossa mostrou-se divergente entre as crianças pesquisadas quanto à $\mathrm{PC}$ unilateral e bilateral.

Tabela 1 - Caracterização sociodemográfica e nível de comprometimento

\begin{tabular}{lcccccc}
\hline & \multicolumn{3}{c}{ Sexo } & Idade & GMFCS \\
\hline Grupo & N & Masculino & Feminino & & Nível I & Nível II \\
PC Unilateral & 10 & $7(70)$ & $3(30)$ & $8,3(2,26)$ & $6(60)$ & $4(40)$ \\
PC Bilateral & 9 & $5(55,55)$ & $4(44,44)$ & $9,1(1,83)$ & $4(44,44)$ & $5(55,55)$ \\
\hline
\end{tabular}

Dados apresentados em n percentil, exceto a idade em média e desvio padrão.

Legenda: PC= Paralisia Cerebral; GMFCS= Classificação da Função Motora Grossa; $n=$ número amostral.

Fonte: Elaborada a partir da coleta dos dados da pesquisa.

Tabela 2 - Funções motoras por distribuição anatômica da paralisia cerebral

\begin{tabular}{lcccccc}
\hline Grupo & A & B & C & D & E & Total \\
\hline Unilateral & 100 & 100 & $98(0,05)$ & $91,79(0,07)$ & $92(0,07)$ & $96(0,03)$ \\
Bilateral & 100 & 100 & 100 & $95,44(0,04)$ & $86,42(0,13)$ & $96(0,03)$ \\
\hline
\end{tabular}

Dados apresentados em média e desvio padrão. Dimensão A: deitar e rolar; Dimensão B: sentar; Dimensão C: engatinhar e ajoelhar; Dimensão D: ficar em pé e Dimensão E: andar, correr e pular. 
Tabela 3 - Habilidades funcionais e assistência do cuidador por distribuição anatômica da PC

\begin{tabular}{lcccccc}
\hline & \multicolumn{3}{c}{ Habilidades Funcionais } & \multicolumn{3}{c}{ Assistência do cuidador } \\
\hline Grupo & AC & MOB & FS & AC & MOB & FS \\
\hline Unilateral & $74,9(13,54)$ & $63,3(5,40)$ & $84,2(13,31)$ & $76,1(12,72)$ & $83,7(14,49)$ & $86,9(19,17)$ \\
Bilateral & $85,3(16,42)$ & $66,1(5,10)$ & $77,8(18,83)$ & $82,4(21,00)$ & $82,4(14,43)$ & $73,8(23,60)$ \\
\hline
\end{tabular}

Dados calculados por escores contínuos, apresentados em média e desvio padrão. Legenda: AC: autocuidado; MOB: mobilidade; FS: função social.

Fonte: Elaborada a partir da coleta dos dados da pesquisa.

As habilidades funcionais das crianças e a necessidade de ajuda do cuidador nas tarefas avaliadas pelo Pedi têm seus resultados apresentados pela Tabela 3, Em que as crianças com PC unilateral obtiveram menores escores na área de autocuidado (AC), tanto na parte de habilidades funcionais quanto na assistência do cuidador.

Os dados para a qualidade de vida das crianças com PC estão apresentados na Tabela 4, na qual se observa por meio de percentual os sete domínios de avaliação do CP QOL-Child. Em todos os sete domínios as crianças com PC unilateral tiveram pontuações inferiores às crianças com PC bilateral, exceto no domínio dor e impacto da deficiência porque se considera para pontuação o nível de dor, observando-se que maior pontuação indica um maior nível de dor nas crianças com PC unilateral.

Tabela 4 - Domínios do CP QOL-Child por distribuição anatômica da PC

\begin{tabular}{lcc}
\hline Domínios & $\begin{array}{c}\text { PC } \\
\text { Unilateral }\end{array}$ & $\begin{array}{c}\text { PC } \\
\text { Bilateral }\end{array}$ \\
\hline Bem-Estar Social e Aceitação & $77,76(0,13)$ & $86,10(0,13)$ \\
Funcionalidade & $68,64(0,17)$ & $78,81(0,15)$ \\
Participação e Saúde Física & $60,45(0,15)$ & $79,67(0,15)$ \\
Bem-Estar Emocional e & $79,58(0,15)$ & $84,49(0,13)$ \\
Autoestima & $74,07(0,17)$ & $79,39(0,14)$ \\
Acesso a Serviços & $35,59(0,19)$ & $30,03(0,16)$ \\
Dor e Impacto da Deficiência & $78,12(0,25)$ & $83,68(0,11)$ \\
Saúde da Família &
\end{tabular}

Dados apresentados em média e desvio padrão. PC= Paralisia Cerebral.

Fonte: Elaborada a partir da coleta dos dados da pesquisa.

\section{DISCUSSÃO}

O estudo observou em seus desfechos que as crianças com PC tanto unilateral quanto bilateral possuem uma qualidade de vida considerada boa na percepção dos pais. As habilidades das crianças, nível de assistência recebida, sugeriram que a distribuição anatômica não foi determinante para definir a funcionalidade das crianças, justificando a variabilidade encontrada no perfil funcional dos resultados.

As crianças com PC unilateral tiveram mais dificuldade para executar a dimensão $C$ (engatinhar e ajoelhar). Esse resultado pode se justificar devido à posição de quatro apoios não ser facilmente executada por essas crianças, pois o hemicorpo comprometido restringe o posicionamento nessa postura. Essas crianças apresentam essa dificuldade no engatinhar, pois o aprendizado anterior a essa tarefa, que se trata do sentar, é uma etapa primordial para que a criança aprenda a se locomover engatinhando ou arrastando seus membros para o engatinhar (DENUCCl; SOUZA, 2019).

Nas crianças com PC unilateral a habilidade funcional de sair da posição sentada para em pé (Dimensão D), usando a posição de joelhos para erguer-se, também é realizada com dificuldade, assim como rolar e sentar. Esse resultado corrobora o fato de que essas crianças necessitam de apoio no hemicorpo comprometido para executar os movimentos, assim como na dimensão $C$ (engatinhar e ajoelhar) também precisa desse apoio para a criança arrastar-se com o membro preservado, locomovendo-se na posição sentada. No geral as crianças com PC apresentam dificuldade no controle do corpo no espaço, para prever ajustes antecipatórios ao realizar atividades e demonstram pouca coordenação, fazendo com que tenham mais dificuldade para se adaptar na realização de atividades cotidianas (PAVÃO et al., 2014).

Para as habilidades de andar, correr e pular (dimensão E) as crianças com PC bilateral obtiveram menor média. Essas crianças apresentam maior comprometimento nos membros inferiores quando comparadas às crianças com PC unilateral, pois essas últimas compensam o movimento com o lado sadio. $\mathrm{Na}$ PC bilateral os sujeitos apresentam insuficiência da capacidade de controle postural e funções motoras alteradas, muito visíveis em tarefas de caminhada e velocidade (MARTINS; FERNANDES; FERREIRA, 2015). Em outro estudo foi encontrado que crianças com PC 
bilateral no desempenho do andar, mudar de posição e manter postura, são situações com determinadas limitações, pois essas habilidades exigem dessas crianças um maior controle motor e postural (SOUZA; ALPINO, 2015). Além disso, estudo destaca que as crianças com PC, mesmo tendo limitações mais leves, apresentam dificuldades relacionadas à capacidade $\mathrm{e}$ à velocidade da marcha para o caminhar (MARTAKIS et al., 2020).

O ambiente em que a criança vive, e os lugares que ela frequenta, tendo ou não apoio social, confirma estar intimamente ligado ao nível de limitação de mobilidade que a criança manifestará no seu desempenho funcional (SOUZA; ALPINO, 2015). As crianças com PC geralmente apresentam distúrbios do movimento e limitações de capacidade, porém neste estudo as crianças apresentaram diferenças apenas em três dimensões, fato que não influenciou na pontuação total da medida tanto na PC bilateral quanto na unilateral.

É importante ressaltar que a pontuação total sem grande variação nos resultados pode ser atribuída ao fato de as avaliações terem ocorrido em ambiente padrão e não no espaço doméstico das crianças. Além disso, vale destacar novamente que as crianças foram classificadas nos níveis I ou II do GMFCS e caminhavam independentemente, sem a utilização de dispositivo manual.

O comprometimento motor e os fatores ambientais estão interligados ao desempenho funcional, pois o meio em que a criança vive e os lugares que frequenta podem oferecer barreiras. Algumas barreiras arquitetônicas ou atitudinais podem atuar como restritoras de participação social das crianças com alguma deficiência, gerando maior isolamento domiciliar e dificuldade em assumir papéis ocupacionais e sociais (JEONG, 2019).

As habilidades e o nível de assistência avaliados pelo Pedi mostraram que as crianças com PC unilateral obtiveram menores escores na área de autocuidado $(A C)$, tanto na parte de habilidades funcionais quanto na assistência do cuidador. Esse achado pode ser justificado pelo fato de as atividades de autocuidado, em sua maior parte, necessitarem do uso das duas mãos para serem realizadas. Nas tarefas bimanuais, o uso das duas mãos deve ser espontâneo, quando a mão dominante executará as manipulações finas e a não dominante estabilizará os objetos, porém quando uma das mãos está comprometida não consegue executar a função esperada (CRICHTON et al., 2020). O dado sugere que essas crianças desprezam o membro comprometido por serem incapazes de utilizá-lo para uso espontâneo em atividades de vida diária, tornando o membro passivo e não funcional.

$O$ fato de as crianças com PC unilateral terem apresentado maior dependência na área de autocuidado pode estar relacionado também com o resultado encontrado na GMFM na dimensão D (ficar em pé), em que os resultados apresentaram-se inferiores na posição em pé. As atividades de autocuidado como banho, escovação de dentes, troca de roupas e pentear os cabelos são atividades habitualmente realizadas em ortostatismo. Esse fato é confirmado quando se considera que a realização do autocuidado necessita do ortostatismo, porém ficar em pé trata-se de uma tarefa complexa, exigindo uma boa retificação postural para alcançá-la (SOUZA, 2019).

$\mathrm{Na}$ área de mobilidade as crianças com PC bilateral tiveram maior escore nas habilidades funcionais, entretanto apresentaram maior grau de dependência de seus cuidadores. Esse resultado pode sugerir que as crianças recebem mais ajuda do que realmente necessitam, pois a postura do cuidador pode limitar a independência, devido ao grau da assistência prestada. As características da família/cuidadores podem apresentar aspectos de proteção, que irão se refletir no desempenho de tarefas diárias.

Quando os cuidadores ofertam um maior nível de auxílio nas tarefas funcionais acabam por gerar uma expectativa inferior nas habilidades de atividades cotidianas, limitando o desempenho, o aprimoramento e consequentemente o desenvolvimento da criança, por não permitirem que a criança desenvolva sozinha suas atividades. A postura dos cuidadores perante o cotidiano das crianças representa muitas vezes a educação parental, a relação com a criança, rotina, personalidade, enfretamento da situação que foi imposta e a maneira como é conduzida. Além disso, as informações da escala de assistência do cuidador no Pedi podem ser influenciadas por suas expectativas e atitudes quando se trata das habilidades funcionais da criança (OLIVEIRA; MATSUKURA; MANCINI, 2015).

Esse resultado também pode ser analisado sob outro olhar, ao levar em consideração que as condições físicas dos ambientes em que essas crianças vivem podem ter restringido o desempenho, de modo a esclarecer essa necessidade de assistência. Estudo já descreveu anteriormente que o ambiente onde a vida é conduzida está diretamente associado com os efeitos que ele pode causar na mobilidade funcional de crianças com PC (KLEINER et al., 2015). 
A postura do cuidador, contudo, e as condições do ambiente podem ter causado implicações nas respostas do Pedi. Esses fatores são claramente influenciadores da participação e envolvimento em situações de vida, acarretando em dificuldades da criança se engajar em atividades sociais e produtivas independentemente. Esse dado referente à área de mobilidade no Pedi está de acordo com o achado na GMFM dimensão $E$ (andar, correr e pular) quando demonstrou que em atividades que envolvam o caminhar as crianças com PC bilateral apresentaram maior comprometimento devido à instabilidade postural e ao controle de tronco, principalmente na PC espástica (MENESES, 2019).

Ao tornar-se mais dependente de seu cuidador em diversas tarefas, entre elas a mobilidade, a criança acaba por ser restringida na sua capacidade de explorar o ambiente e de estabelecer vínculos sociais com outras pessoas (PAVÃO et al., 2014). O ambiente escolar é um dos locais em que as crianças têm maior grau de comprometimento da função social, pois a participação em jogos e brincadeiras pode ser prejudicada devido às limitações motoras, cognitivas e de mobilidade. A socialização com outras crianças torna-se deficitária, diminuindo o envolvimento em atividades de ensino e recreação, levando ao isolamento.

Além da interação no ambiente escolar, a socialização em comunidade também é um aspecto que irá predizer sobre as habilidades sociais de crianças com PC, pois as relações interpessoais, interação com outras crianças e adultos, assim como o enfrentamento na resolução de problemas tornam o sujeito socialmente engajado e empoderado. Quando não ocorre participação social a criança fica mais retraída, dependente de seus cuidadores em momentos de trocas com outras pessoas, o que poderá levar a diversos outros problemas no cotidiano. Um estudo afirma que a criança com falta de participação social geralmente terá problemas em seu comportamento (LOPES; MATSUKURA, 2018).

Na avaliação CP QOL-Child faz-se importante ressaltar que o domínio da dor e impacto da deficiência do CP QOL-Child caracterizam-se por questões indicativas do nível de dor que a criança sente e se fica chateada ou desconfortável com situações referentes à PC. Nesse domínio citado a pontuação também é de zero a nove, no entanto zero representa nenhum desconforto e nove significa muito desconforto. Dessa forma, quanto menor a pontuação obtida melhores são os resultados referentes a esse domínio, o que justifica a pontuação muito inferior quando comparado aos demais domínios do instrumento.
A partir da apreciação do CP QOL-Child entende-se que ele precisa ser analisado isoladamente, pois não há possibilidade de estabelecer um paralelo com os demais instrumentos do estudo, visto que a avaliação da qualidade de vida é multidimensional e subjetiva, nos demais instrumentos utilizados neste estudo obtém-se resultados mais objetivos do que os resultados para qualidade de vida. Dessa forma, os dados desse instrumento são interpretados e discutidos dentro da dimensão da subjetividade, considerando que não é possível aferir instrumentos subjetivos junto com instrumentos objetivos.

A avaliação da qualidade de vida de crianças com PC, sob a ótica dos pais ou cuidadores, pode sofrer influências de fatores relacionados a questões de ordem pessoal e emocional. 0 estresse e a sobrecarga dos pais, bem como a dificuldade com as estratégias de enfrentamento da situação do filho, podem afetar os dados da qualidade de vida. Além disso, crianças com PC apresentam dificuldade em participar de atividades em casa, na escola e na comunidade em geral, como resultado de restrições, que podem levar os pais a relatarem uma qualidade de vida reduzida para seu filho (CALLEY et al., 2012).

O nível de compreensão dos pais e a forma de interpretar as perguntas também são aspectos a serem considerados ao avaliar esses resultados, pois o entendimento das perguntas pode sugerir estilos de respostas distintas das que o entrevistado daria ao pesquisador. Entre os itens avaliados no CP QOL-Child estão o bem-estar social, emocional e autoestima, que são áreas puramente subjetivas e dependentes de crenças, contextos de vida e experiências familiares. Dessa forma, cada pessoa pode apresentar diferentes experiências, de acordo com o modo de entender e conduzir sua vida em seus contextos de inclusão ou até de participação social, seja ela limitada ou ativa para a deficiência apresentada.

Neste estudo foi utilizado um instrumento de qualidade de vida específico para PC, versão questionário do cuidador primário, ou seja, respondido pelos pais, responsável ou cuidador principal da criança. A literatura apresenta alguns estudos sobre a qualidade de vida por ser tratar de crianças com PC, mostrando maior dificuldade em avaliar a própria perspectiva da criança quanto à qualidade de vida, devido a aspectos limitadores, como a idade, dificuldades na fala ou de compreensão cognitivas (BRACCIALLI; RIBEIRO; BRACCIALLI, 2019).

De acordo com a literatura consultada, esta é a primeira pesquisa que associou os instrumentos GMFM, Pedi e CP-QOL Child para avaliar a funciona- 
lidade de crianças com PC no componente atividade e participação da CIF. Utilizou-se a GMFM para avaliar as capacidades das crianças em atividades de função motora ampla em ambiente padrão, mais especificamente na área de mobilidade. O Pedi e o CP QOL-Child foram utilizados para avaliar, a partir da perspectiva dos pais, responsável ou cuidador, o desempenho das crianças em tarefas cotidianas em seu ambiente real. Estes revelaram aspectos referentes à aprendizagem, comunicação, autocuidado, vida doméstica, relacionamentos interpessoais e relações sociais.

Este estudo fornece informações importantes sobre a avaliação de crianças com PC com base no modelo da CIF de abordagem biopsicossocial. A investigação das capacidades dessas crianças e do seu desempenho funcional pode contribuir no planejamento e nas estratégias de intervenção em crianças com PC, a partir de uma avaliação estruturada, com caráter abrangente de saúde, pois considera as habilidades funcionais das crianças e a qualidade de vida. As principais limitações do estudo foram a entrevista em ambiente padrão, em que não foi possível compreender melhor o ambiente domiciliar em que a criança vive, $\mathrm{e}$ as limitações e barreiras arquitetônicas do ambiente. Outro fator foi a amostra reduzida, pois muitas crianças não se enquadraram nos critérios de inclusão.

\section{CONCLUSÃO}

O estudo propiciou a compreensão de forma ampliada sobre a funcionalidade e qualidade de vida das crianças baseada nos componentes atividade e participação na CIF, explorando várias situações diárias que a criança executa e como se caracteriza a sua independência. Os três instrumentos utilizados mostraram-se importantes, fornecendo uma melhor compreensão das atividades (capacidade) e participação (desempenho) em situações de vida, de modo que contemplaram todas as áreas vitais do componente em estudo.

As equipes de saúde devem ampliar o olhar sobre o indivíduo, considerando as funções e estruturas corporais, mas também analisar aspectos relacionados às atividades e participação, assim como os fatores pessoais e ambientais que interferem diretamente na funcionalidade das crianças, aplicando as orientações para a realidade social em que as crianças com alguma deficiência vivenciam.

Os resultados deste estudo apontaram que na GMFM as crianças com PC bilateral e unilateral apresentaram o mesmo nível de função motora ampla. No Pedi os dados mostraram-se diversos para as crianças e no CP QOL-Child as crianças com PC bilateral tiveram maiores escores. Esses dados sugeriram que a distribuição anatômica da PC em bilateral e unilateral não foi determinante para definir a funcionalidade das crianças, justificando a variabilidade encontrada no perfil funcional. Os fatores contextuais (físicos, sociais e atitudinais) manifestaram influência substancial na funcionalidade dessas crianças, seja de forma positiva (facilitador) ou negativa (barreira), uma vez que as condições físicas dos ambientes que os participantes frequentam, assim como as atitudes dos cuidadores, apareceram como aspectos principais.

Sugere-se para estudos futuros a replicação com um tamanho de amostra maior, pois o restrito número do grupo amostral desta pesquisa caracterizou-se como uma limitação. A mensuração da GMFM no ambiente real da criança também pode ser um importante aspecto a ser considerado nos estudos posteriores a este.

\section{ABREVIATURAS E SIGLAS}

CIF - Classificação Internacional de Funcionalidade, Incapacidade e Saúde.

CP QOL-Child - Questionário de Qualidade de Vida de Crianças com PC

DP - Desvio padrão

GMFCS - Sistema de Classificação da Função Motora Grossa

GMFM - Medida de Função Motora Grossa

OMS - Organização Mundial da Saúde

PC - Paralisia Cerebral

PEDI - Inventário de Avaliação Pediátrica de Incapacidade

UFSM - Universidade Federal de Santa Maria

\section{REFERÊNCIAS}

BIZ, M. C. P.; CHUN, R. Y. S. Operacionalização da Classificação Internacional de Funcionalidade, Incapacidade e Saúde, CIF, em um Centro Especializado em Reabilitação. Revista CoDAS, São Paulo, v. 32, n. 2, e20190046, 2019.

BRACCIALLI, A. C.; RIBEIRO, A. S.; BRACCIALLI, L. M. P. Qualidade de vida de crianças com paralisia cerebral não deambuladoras: autorrelato. In: SEMINÁRIO DO GRUPO DE PESQUISA DEFICIÊNCIAS FÍSICAS E SENSORIAIS DA FACULDADE DE FILOSOFIA E CIÊNCIAS, 5., Anais [...]. Faculdade de Filosofia e Ciências, 2019. p. 41-48.

BRACCIALLI, L. M. P. et al. Questionário de qualidade de vida de crianças com paralisia cerebral (CP QOL - CHILD): tradução e adaptação para a língua portuguesa. Journal of Human Growth and Development, v. 23, n. 2, p. 154-163, 2013. 
BRASIL. Ministério da Saúde. Estatuto da Criança e do Adolescente. 6. ed. Brasília, 2005. Disponível em: http://www. planalto.gov.br/ccivil_03/leis/L8069.htm.

BRITTO, H. M. J. S. et al. Contextual factors associated with life-space mobility in community-dwelling older adults based on International Classification of Functioning, Disability and Health: protocol for a systematic review. BMJ Open, v.8, n.10, 2018.

CALLEY, A. et al. A comparison of activity, participation and quality of life in children with and without spastic diplegia cerebral palsy. Disabilities \& Rehabilitation, v. 34, n. 15, p. 1.306-1.310, 2012.

CRICHTON, A. et al. Brain magnetic resonance imaging is a predictor of bimanual performance and executive function in children with unilateral cerebral palsy. Developmental Medicine \& Child Neurology, v. 62, ed. 5, 2020.

CYRILLO, L. T.; GALVÃO, M. C. S. Medida da função motora grossa: [GMFM-66 \& GMFM-88]: manual do usuário. 1. ed. São Paulo: Ed. Memnon, 2011.

CYRILLO, L. T.; GALVÃO, M. C. S. Medida da função motora grossa: [GMFM-66 \& GMFM-88]: manual do usuário. 2. ed. São Paulo: Ed. Memnon, 2015.

DAVIS, E. et al. Measuring the quality of life of children with cerebral palsy: comparing the conceptual differences and psychometric properties of three instruments. Developmental Medicine \& Child Neurology, v. 52, n. 2, p. 174-180, 2010.

DENUCCI, M. A. M.; SOUZA, C. H. M. A linguagem na criança com encefalopatia crônica da infância. In: XIV Jornada Nacional de Linguística e Filologia de Língua Portuguesa... Anais, ano 25, n. 75 Supl., 2019.

DORNELES, L. F. et al. Aplicabilidade da Classificação Internacional de Funcionalidade, Incapacidade e Saúde (CIF) para a avaliação de crianças com paralisia cerebral: uma revisão sistemática. Cadernos de Terapia Ocupacional da UFSCar, v. 22, n. 3, p. 579-590, 2014.

FLUSS, J.; LIDZBA, K. Cognitive and academic profiles in children with cerebral palsy: a narrative review. In: Annals of Physical and Rehabilitation Medicine, 2020.

HIRATUKA, E.; MATSUKURA, T. S.; PFEIFER, L. I. Adaptação transcultural para o Brasil do sistema de classificação da função motora grossa (GMFCS). Revista Brasileira de Fisioterapia, v. 14, n. 6, p. 537-544, 2010.

JEONG, Y. Participation, supports, and barriers of Korean children and youth with and without disabilities in the school environment. Disability Rehabilitation, v. 3, p. 1-8, 2019.

KLEINER, A. F. R. et al. Context-dependency of mobility in children and adolescents with cerebral palsy: optimal and natural environments. Journal of Policy Practice Intellectual Disabilities, v. 12, n. 4, p. 288-293, 2015.

LOPES, P.; MATSUKURA, T. Perspectivas futuras sobre participação social na ótica de adolescentes com paralisia cerebral e suas mães. Revista de Terapia Ocupacional da Universidade De São Paulo, v. 2, n. 1, p. 19-26, 2018.
MANCINI, M. C. Inventário de avaliação pediátrica de incapacidade (PEDI). Manual da versão brasileira adaptada. Belo Horizonte: UFMG, 2005.

MARTAKIS, K. et al. Teste de caminhada de um minuto em crianças com paralisia cerebral GMFCS nível 1 e 2: valores de referência para identificar efeitos terapêuticos após reabilitação, Neurorreabilitação do Desenvolvimento, v. 23, n. 4, p. 201-209, 2020.

MARTINS, E.; FERNANDES, J.; FERREIRA, A.C. Movement patterns during the process of standing up in children with spastic diplegia. Motriz, Rio Claro, v. 21, n. 3, p. 230-236, July/Sept. 2015.

MENESES, D. C. S. Avaliação funcional de tronco e membros superiores de crianças espásticas tratadas com cinesioterapia e ziclague. 2019. 75 p. Dissertação (Mestrado em Saúde e Ambiente) - Universidade Tiradentes, Aracaju, 2019.

OLIVEIRA, A. K. C.; MATSUKURA, T. S.; MANCINI, M. C. Repertório funcional de crianças com paralisia cerebral nos contextos domiciliar e clínico: relato de cuidadores e profissionais. Revista de Terapia Ocupacional da Universidade de São Paulo, v. 26, n. 3, p. 390-398, 2015.

PAVÃO, S. L. et al. Relationship between static postural control and the level of functional abilities in children with cerebral palsy. Brazilian Journal of Physical Therapy, v. 18, n. 4, p. 300-307, 2014.

PIEXAK, D. R. et al. Classificação Internacional de Funcionalidade, Incapacidade e Saúde: uma análise de conteúdo. Journal of Research: Fundamental Care On-line, v. 11, n. especial, p. 363-369, 2019.

SCHIARITI, V. et al. Comparing contents of outcome measures in cerebral palsy using the international classification of functioning (ICF-CY): A systematic review. European Journal of Pediatric Neurology, v. 8, n. 1, p. 1-12, 2013.

SCHIARITI, V.; MÂSSE, L. C. Relevant areas of functioning in children with Cerebral Palsy based on the International Classification of Functioning, Disability and Health Coding System: A clinical perspective. Journal of Child Neurology, v. 30, n. 2, p. 2.016-2.022, 2015.

SILVA, D. B. R.; DIAS, L. B.; PFEIFER, L. I. Confiabilidade do Sistema de Classificação da Função Motora Grossa Ampliado e Revisto (GMFCS E \& R) entre estudantes e profissionais de saúde no Brasil. Revista Fisioterapia e Pesquisa, v. 23, n. 2, p. 142-7, 2016

SILVA, D. B. R.; PFEIFER, L. I.; FUNAYAMA, C. A. R. Sistema de classificação da função motora grossa ampliado e revisto (GMFCS E \& R), 2010. Disponível em: https://canchild.ca/ system/tenon/assets/attachments/000/000/075/original/ GMFCS-ER_Translation-Portuguese2.pdf. Acesso em: 24 mar. 2020.

SOUZA, A. C. S. O processo de levantar em crianças e adolescentes com paralisia cerebral: análise de padrão dos padrões de movimento. 2019. 111 p. Dissertação (Mestrado em Educação Física) - Universidade de Brasília, Brasília, 2019. 
SOUZA, N. P.; ALPINO, A. M. S. Avaliação de crianças com diparesia espástica segundo a Classificação Internacional de Funcionalidade, Incapacidade e Saúde (CIF). Revista Brasileira de Educação Especial, v. 21, n. 2, p. 199-212, 2015.

WAGNER, D. et al. Desenvolvimento de dispositivo de sustentação e movimentação para a cabeça de crianças com paralisia cerebral espástica. Brazilian Journal of Development, Curitiba, v. 6, n. 3, p. 10.088-10.105, 2020. 